\title{
Jupiter and the other Giants: A Comparative Study
}

\author{
Thérèse Encrenaz \\ LESIA, Observatoire de Paris \\ F-92190 Meudon, France \\ email: therese.encrenaz@obspm.fr
}

\begin{abstract}
The four giant planets - Jupiter, Saturn, Uranus and Neptune - have common properties which make them very different from the terrestrial planets: located at large distances from the Sun, they have big sizes and masses but low densities; they all have a ring system and a large number of satellites. These common properties can be understood in the light of their formation scenario, based upon the accretion of protosolar gas on an initial icy core. Giant planets have been explored by space missions (Pioneer 10 and 11, Voyager 1 and 2, Galileo and Cassini) but also by Earth-orbiting satellites and ground-based telescopes. There are still open questions related to the origin and evolution of the giant planets, in particular their moderate migration, the origin of the cold planetesimals which formed Jupiter, the origin of the atmospheric dynamics in Jupiter and Saturn, and the differences in the internal structures of Uranus and Neptune.
\end{abstract}

Keywords. Planets and satellites: formation, Planets and satellites: general; Solar system: formation

\section{Introduction}

The four giant planets - also called jovian planets - are located in the outer solar system, at heliocentric distances ranging between 5 and 30 AU. As shown in Table 1 and 2 , their orbital and physical properties (mass, radius, density), as well as their rings and satellite systems make them very different from the terrestrial planets (Mercury, Venus, the Earth and Mars) which all orbit within 2 AU from the Sun.

\section{Early observations}

Jupiter and Saturn, as naked-eye objects, have been known since Antiquity. However, their physical properties remained unknown until Galileo, in 1610, started to look at them with his new telescope. His major discovery was the identification of four satellites around Jupiter (the "Medicean moons", later renamed galilean satellites), which came in support of the heliocentric system proposed a few decades earlier by Copernic. In addition to other spectacular results - the relief on the Moon, the phases of Venus, the multitude of stars which populate the Milky Way -, Galileo also observed the changing aspect of Saturn's disk, depending on the orientation of its rings with respect to the Earth, but could not identify the explanation of this variation. The answer was given in 1659 by Christiaan Huygens, who also discovered Saturn's largest satellite Titan. With the apparition of large observatories at the end of the $\mathrm{XVII}^{\text {th }}$ century, astronomical observations of Jupiter and Saturn were made on a regular basis. Cassini monitored the Great Red Spot of Jupiter and its system of zones and bands; he identified a gap in Saturn's rings, later called Cassini's Division, and discovered several of Saturn's icy satellites. 
Table 1. Orbital properties of solar-system planets.

\begin{tabular}{lcccc}
\hline Name & $\begin{array}{c}\text { Semi-major } \\
\text { axis (AU) }\end{array}$ & Eccentricity & $\begin{array}{c}\text { Inclination over } \\
\text { the Ecliptic }\left(^{\circ}\right)\end{array}$ & $\begin{array}{c}\text { Revolution period } \\
\text { (years) }\end{array}$ \\
\hline $\begin{array}{l}\text { Terrestrial } \\
\text { Planet }\end{array}$ & & & & \\
Mercury & 0.39 & 0.205 & 7.00 & 0.241 \\
Venus & 0.72 & 0.007 & 3.39 & 0.615 \\
Earth & 1.00 & 0.017 & 0.00 & 1.000 \\
Mars & 1.52 & 0.093 & 1.85 & 1.881 \\
Giant & & & & \\
Planet & & & & \\
Jupiter & 5.20 & 0.054 & 1.30 & 11.856 \\
Saturn & 9.54 & 0.047 & 2.48 & 83.747 \\
Uranus & 19.2 & 0.086 & 0.77 & 163.723 \\
Neptune & 30.1 & 0.008 & 1.77 & \\
\hline
\end{tabular}

Table 2. Physical properties of solar-system planets.

\begin{tabular}{lccccc}
\hline Name & Mass $\left(\mathbf{M}_{E}\right)$ & $\begin{array}{c}\text { Equatorial } \\
\text { radius }\left(\mathbf{R}_{E}\right)\end{array}$ & $\begin{array}{c}\text { Density } \\
\left(\mathrm{g} / \mathbf{c m}^{3}\right)\end{array}$ & $\begin{array}{c}\text { Rotation } \\
\text { period }\end{array}$ & Obliquity $\left(^{\circ}\right)$ \\
\hline $\begin{array}{l}\text { Terrestrial } \\
\text { Planet }\end{array}$ & & & & & \\
Mercury & 0.055 & 0.382 & 5.43 & $58.646 \mathrm{~d}$ & 0.0 \\
Venus & 0.815 & 0.949 & 5.20 & $243.018 \mathrm{~d}$ & 177.33 \\
Earth & 1.000 & 1.000 & 5.52 & $23.934 \mathrm{~h}$ & 23.45 \\
Mars & 0.107 & 0.532 & 3.93 & $24.623 \mathrm{~h}$ & 25.19 \\
Giant & & & & & \\
Planet & & & & & \\
Jupiter & 317.9 & 11.21 & 1.33 & $9.925 \mathrm{~h}$ & 3.08 \\
Saturn & 95.16 & 9.45 & 0.69 & $10.656 \mathrm{~h}$ & 26.73 \\
Uranus & 14.53 & 4.00 & 1.32 & $17.24 \mathrm{~h}$ & 97.92 \\
Neptune & 17.14 & 3.88 & 1.64 & $16.11 \mathrm{~h}$ & 28.80 \\
\hline
\end{tabular}

At the end of the XVIII ${ }^{t h}$ century, a new step was achieved in planetary exploration with the discovery of a new planet beyond Saturn. In 1781, William Herschel, using a larger telescope of improved quality, detected an unknown object which was called Uranus, thus extending the frontiers of the solar system. At the same time, a new science, celestial mechanics, was developing rapidly, on the basis of Newton's law of universal gravitation. In 1821, precise calculations of the giant planets' orbits showed evidence for an anomaly in Uranus' orbit; the only possible explanation had to be the orbit's gravitational perturbation by a more distant planet. Two astronomers, John Couch Adam in England and Urbain Le Verrier in France, independently found the coordinates of the object. Le Verrier sent the coordinates of the new planet to Johannes Galle who immediately found it within $1^{\circ}$ of its predicted position. The discovery of this new planet, called Neptune, marked the triumph of celestial mechanics. 


\section{The space exploration}

Giant planets were first explored by two NASA-led missions in the 1970s, first by the Pioneer 10 and 11 spacecraft, then by the Voyager mission. Pioneer 10 encountered Jupiter in 1973; Pioneer 11 encoutered Jupiter in 1974 and Saturn in 1979. The Pioneer mission provided us with the first amazing images of Jupiter's meteorology and Saturn's rings, and with the first exploration of the planets' magnetospheres. Soon after Pioneer, the very ambitious and successful Voyager mission has been a major milestone in the space exploration of the giant planets. Two identical spacecraft, Voyager 1 and Voyager 2, successively encountered the four giant planets between 1979 and 1989. Voyager 1 flew by Jupiter in 1979 and Saturn in 1980, with a close approch of Titan; Voyager 2 flew by Jupiter in 1979, Saturn in 1981, Uranus in 1986 and Neptune in 1989. Among the Voyager highlights are the discoveries of Io's volcanism, Europa's probable subsurface water ocean, the complex structure of Saturn's rings, Miranda's traces of tectonic activity, Triton's cryovolcanism, and the complex structure of the giant planets' magnetospheres.

Ten years later, the Galileo mission was launched by NASA for an in-depth exploration of the jovian system. On December 7, 1995, a descent probe entered Jupiter's atmosphere and transmitted data down to a pressure level of 22 bars. An orbiter performed an in-depth exploration of the planet and the galilean satellites from 1995 to 2003. Ten years later, Saturn's system was approached by the Cassini mission, launched in 1997 by NASA in collaboration with ESA. On January 14, 2005, the Huygens probe, led by ESA, successfully landed on Titan's surface and provided the world with the first images of Saturn's biggest satellite. The Cassini orbiter, in operation since 2005, is planned to continue its exploration of Saturn's system until 2017.

In addition to in-situ planetary exploration, our knowledge of the giant planets has benefited from Earth-orbit observations : the International Ultraviolet Explorer (IUE) and the Hubble Space Telescope (HST) in the UV, the HST in the visible, the Infrared Space Observatory (ISO) and Spitzer in the IR, and more recently Herschel in the far-infrared and submillimeter range. Infrared and millimeter ground-based observations have also been precious for investigating the chemical composition of the giant planets.
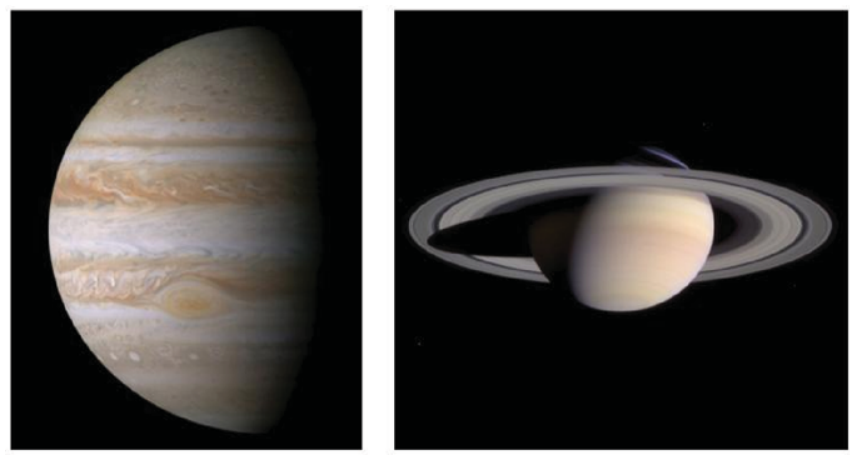

Figure 1. Jupiter (left) and Saturn (right) as observed by the Cassini spacecraft in 2000 and 2004 respectively ((c) NASA). 


\section{The giant planets' formation scenario}

Why do we have two distinct classes of planets in the solar system? The answer can be found in the formation scenario of the solar system, now widely accepted in the scientific community. This scenario is based on a few simple observations: all planets rotate in the same direction around the Sun on nearly circular, coplanar and concentric orbits. These facts led to the concept of the Primordial Nebula model, proposed in the XVIII ${ }^{t h}$ century, first by Immanuel Kant and later by Pierre-Simon de Laplace : a fragment of interstellar cloud collapses into a disk, as a result of its own gravity and rotation. At the center, matter accretes to form the young Sun while, within the turbulent disk, planets form from the accretion of solid particles. This scenario, observed on a number of nearby young stars and protoplanetary disks, is believed to be common the Universe; it is even more supported with the discovery of several hundreds extrasolar planets over the past fifteen years. Particles gather into small embryos as an effect of turbulence and multiple collisions, and accrete into km-size planetesimals; later, the largest cores sweep the surrounding material and grow by gravity. Numerical simulations show that a small number of planet-sized objects can emerge within a few million years or less.

Among the protosolar disk, the size of the planet finally depends upon the amount of solid material available to build the core; this is where the difference between terrestrial and giant planets appears. The protosolar disk is mostly composed of light elements (hydrogen and helium). The abundances of other elements $(\mathrm{C}, \mathrm{N}, \mathrm{O}, \ldots)$ follow the cosmic values, i.e. the heaviest ones are the least abundant. Near the Sun, the temperature is high enough for the simple molecules $\left(\mathrm{H}_{2} \mathrm{O}, \mathrm{CH}_{4}, \mathrm{NH}_{3}, \mathrm{CO}_{2}, \mathrm{H}_{2} \mathrm{~S} \ldots\right)$, most abundant after $\mathrm{H}_{2}$ and $\mathrm{He}$, to be gaseous. The only solid material is thus made of silicates and metals. As their cosmic abundance is small, only small and dense objects can be formed: they are the terrestrial planets. In contrast, at larger heliocentric distances, where the temperature is below about $200 \mathrm{~K}$, the simple molecules mentioned above are in the form of ices and big cores can be formed. Models predict that, when a critical mass of about 10-15 terrestrial masses is reached, their gravity is sufficient for the surrounding nebula (mostly made of gaseous hydrogen and helium) to collapse on them and to form a sub-disk along the cores' equatorial planes. As a result, big planets of low density are formed: the giant planets. Within the sub-disks, solid matter accretes into several bodies: the regular satellites of the giant planets. Ring systems are found in the immediate
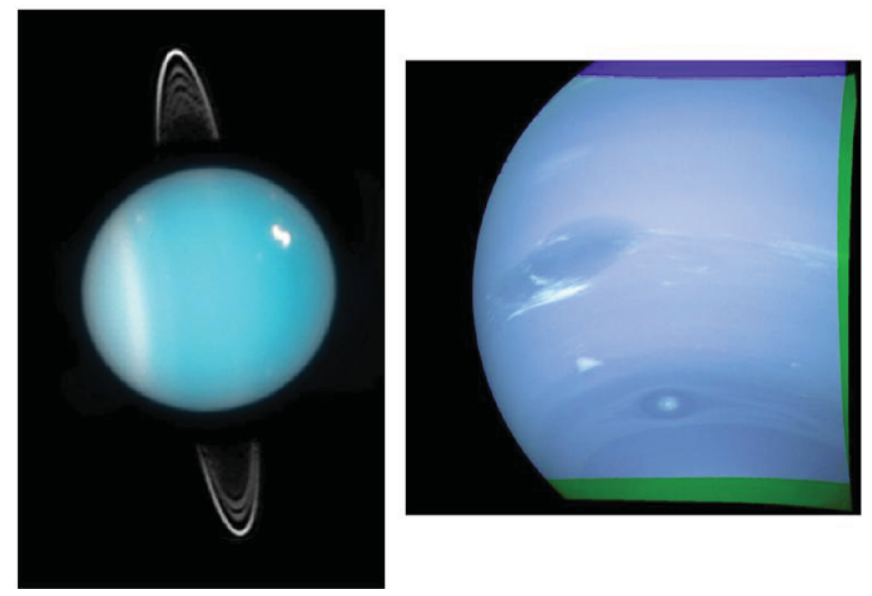

Figure 2. Left: Uranus, as observed by the HST in 2005. Right: Neptune, as observed by Voyager 2 in 1989 ((c)NASA) 
proximity of the planets, within the Roche limit, where tidal forces prevent the accretion of solid bodies larger than planetesimals. The limit beween the terrestrial planets and the giant planets is called the "snowline" which corresponds to the distance of condensation of the ices. Water plays a major role for two reasons: first, being made of two abundant atoms, $\mathrm{H}$ and $\mathrm{O}$, it is the most common ice; second $\mathrm{H}_{2} \mathrm{O}$ is the first molecule to condense as the temperature decreases; other ices $\left(\mathrm{NH}_{3}, \mathrm{CO}_{2}, \mathrm{H}_{2} \mathrm{~S}, \mathrm{CH}_{4} \ldots\right)$ condense at larger heliocentric distances. This explains the composition of outer satellites, mostly made of water ice.

Table 2 shows that the giant planets fall into two categories. Jupiter and Saturn, with masses close to 300 and 100 terrestrial masses, are mostly made of protosolar gas; they are called the gaseous giants. In contrast, Uranus and Neptune, with masses of about 15 terrestrial masses, are mostly made of their icy core; they are called the icy giants. What is the origin of this difference? A plausible explanation might be that Uranus and Neptune, formed at larger heliocentric distances, needed more time to accrete their icy core, possibly more than 10 million years. The critical mass of 10 terrestrial masses might have been reached after the dissipation of the protosolar gas. Indeed, the observation of nearby protoplanetary disks shows that their lifetime is usually shorter than $10 \mathrm{My}$.

The study of protoplanetary disks and extrasolar planets also suggests that many giant exoplanets, found close to their parent star, have encountered inward migration, as a result of their interaction with the turbulent disk. Recent dynamical calculations, performed at the Nice Observatory by A. Morbidelli and his colleagues, suggest that in the solar system, giant planets exhibited a moderate migration, with Jupiter moving inward and the three other giants moving outward. A signature of this migration appears to be the Late Heavy Bombardment (LHB), observed on the surfaces of all bare solar-system objects as a strong maximum of impact craters dated at -3.8 Gy, i.e. about 800 My after the planets' formation. Numerical simulations show that, at that time, the Jupiter-System may have crossed the 2:1 resonance (Saturn's revolution period being twice Jupiter's one), which must have led to a huge perturbation in the orbits of small bodies, with high eccentricities and inclinations. Now, why was the giant planets' migration moderate in the solar system, while it appears to be so efficient around nearby stars? This question is still open.

\section{Atmospheric composition and structure}

The thermal structure of the giant planets shows different regions. In the convective trospophere, the temperature decreases as the altitude increases, following the adiabatic gradient, up to the tropopause where the temperature is minimum. The pressure level at the tropopause is about 100 mbar for all giant planets; the temperature at this level is $110 \mathrm{~K}$ for Jupiter, $90 \mathrm{~K}$ for Saturn, and about $50 \mathrm{~K}$ for Uranus and Neptune. In the stratosphere, above the tropopause, the temperature increases again with altitude, due to the absorption of solar radiation by methane and aerosols. In this region, the photodissociation of methane leads to the formation of several hydrocarbons $\left(\mathrm{C}_{2} \mathrm{H}_{2}, \mathrm{C}_{2} \mathrm{H}_{6} \ldots\right)$. In the hydrogen-dominated atmospheres of the giant planets, most of the species are in reduced form. Tropospheric species include $\mathrm{CH}_{4}, \mathrm{NH}_{3}, \mathrm{H}_{2} \mathrm{O}, \mathrm{PH}_{3}, \mathrm{GeH}_{4}$ and $\mathrm{AsH}_{3}$. In the case of Uranus and Neptune, only methane is observed, because the condensation levels of the other species are too deep to be observable. In 1997, the ISO satellite has detected oxygen species in the stratospheres of all giant planets $\left(\mathrm{H}_{2} \mathrm{O}, \mathrm{CO}_{2}\right)$. The origin of this external oxygen source might be either local (rings, satellites) or interplanetary (comets, micrometeorites). CO has also been detected; its origin (internal or external, or both) is still a matter of debate. In the case of Neptune, unexpected stratospheric 
abundances of $\mathrm{CO}$ and $\mathrm{HCN}$ have been found; a possible origin might be a cometary impact. Such event (the collision of comet Shoemaker-Levy 9 with Jupiter in 1994) is believed to be at least partly responsible for the presence of the stratospheric water observed during the following years, as well as the CO and HCN high stratospheric contents. The cloud structure of the giant planets can be studied by the images of the planetary disks. The Great Red Spot, identified on Jupiter since the XVII ${ }^{t h}$ century, is believed to be a giant anticyclonic feature; its stability over such a long time is still not fully understood. Saturn is also dynamically very active, with huge cyclones at both poles and a peculiar hexagonal convective feature around the north pole. The mechanism of such activity is still an open problem. The alternative structure in zones and belts, easily observed on Jupiter and Saturn, is the signature of a Hadley-type convective circulation in a fast rotating gaseous sphere. The zones are cloudy regions of ascending motions and the belts are dry regions of subsidence. Additional information on the cloud structure of giant planets is given by models of thermochemical equilibrium. In the case of Jupiter and Saturn, a $\mathrm{NH}_{3}$ cloud is expected (and indeed observed as a white-yellow layer) at a temperature level of $145 \mathrm{~K}(\mathrm{P}=0.5-1$ bar $)$. Clouds of $\mathrm{NH}_{4} \mathrm{SH}(\mathrm{T}=210 \mathrm{~K})$ and $\mathrm{H}_{2} \mathrm{O}(\mathrm{T}=270 \mathrm{~K})$ are expected at deeper levels. In the case of Uranus and Neptune, the temperature is low enough for $\mathrm{CH}_{4}$ to condense at a level of about 1 bar $(\mathrm{T}=80 \mathrm{~K})$. A $\mathrm{H}_{2} \mathrm{~S}$ cloud is expected at about 3 bars $(\mathrm{T}=120 \mathrm{~K})$, and clouds of $\mathrm{NH}_{4} \mathrm{SH}$ and $\mathrm{H}_{2} \mathrm{O}$ are expected at deeper levels. In the stratosphere, hydrocarbon aerosols are expected, coming from the methane photolysis.

In the case of Jupiter, the Galileo probe, in 1995, entered one of the dry "hot spots" which probe the deep troposphere. Elemental abundance ratios were mesured by mass spectrometry for several elements. For six of them (C, N, S, Ar, Xe, Kr) an enrichment by a factor 4 with respect to hydrogen was mesured, as compared with the protosolar values. This enrichment was found to be in full agreement with the predicted values derived from the nucleation model of the giant planets, assuming an initial icy core of 12 terrestrial masses. This result, however, raised an unexpected problem. The uniform enrichment by a factor 4 implies that all the measured elements were equally trapped in

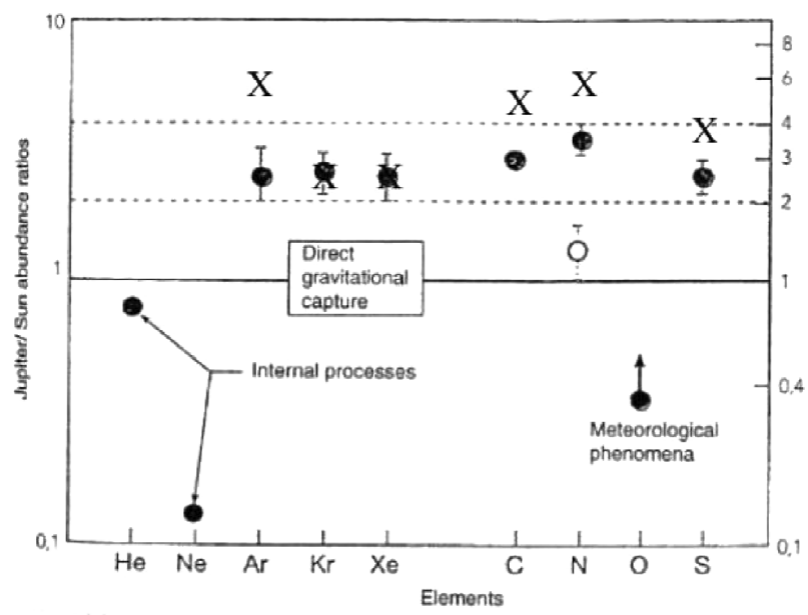

Figure 3. Enrichments of heavy elements in Jupiter as measured by the Galileo Probe Mass Spectrometer. The crosses indicate updated values, following a reestimation of the solar abundances. He and Ne are believed to be depleted by condensation within the metallic hydrogen of Jupiter's interior. $\mathrm{O}$ is depleted in the hot spot that the probe explored by convective motions. The figure is adapted from Owen et al. (1999) and Owen and Encrenaz (2006).) 
ices. Still, this is not expected for Ar and N unless the temperature is very low $(<40 \mathrm{~K})$, much lower than the expected temperature at Jupiter's orbit (about $110 \mathrm{~K}$ ). What is the origin of the cold planetesimals which formed Jupiter? This is still an open question.

What is the situation for the other giants? Unfortunately, we have no in-situ measurement; however, $\mathrm{C} / \mathrm{H}$ is known from $\mathrm{CH}_{4}$ measurements, and here again, the measured $\mathrm{C} / \mathrm{H}$ ratio is in full agreement with the predictions of the nucleation model: an enrichment factor of 9 for Saturn and about 30 for Uranus and Neptune. This conclusion is also supported by the measurement of the $\mathrm{D} / \mathrm{H}$ ratio in the giant planets. This ratio, which is enriched in ices at low temperatures, is indeed diagnostic of the temperature formation of these objects. $\mathrm{D} / \mathrm{H}$ in the giant planets has been measured from the observation of HD infrared lines by ISO. D/H in Jupiter and Saturn was found to be consistent with its protosolar value $\left(2.110^{-5}\right)$, which was expected as these planets are mostly made of protosolar gas. In contrast, $\mathrm{D} / \mathrm{H}$ in Uranus and Neptune was found to be enriched by a factor of about 2.5, in agreement again with the core accretion model. What is the temperature formation of the planetesimals which formed Saturn, Uranus and Neptune? The answer will be given by future space missions, when descent probes are sent into their tropospheres.

\section{Similarities and differences among the giant planets}

With comparable chemical compositions, one would expect the spectra of the gaseous giants (Jupiter and Saturn) to be similar, as well as those of the icy giants (Uranus and Neptune). It is indeed the case of the near-infrared solar component (1-3 $\mu \mathrm{m})$ where all spectra are dominated by methane, a very active spectroscopic agent. In the midinfrared thermal range, spectra show significant differences which are the signature of intrinsic differences in their atmospheric dynamics. A first example is shown in Figure 4 , in the 7-12 $\mu \mathrm{m}$ range. The spectra of Jupiter and Saturn show drastic differences : in particular, a strong $\mathrm{NH}_{3}$ band dominates the Jovian spectrum while Saturn's exhibits $\mathrm{CH}_{3} \mathrm{D}$ and $\mathrm{PH}_{3}$ signatures. There are two main reasons for these differences. The first is the colder environment of Saturn, where ammonia is mostly condensed in clouds. The second reason is a higher vertical dynamical activity on Saturn. This is illustrated

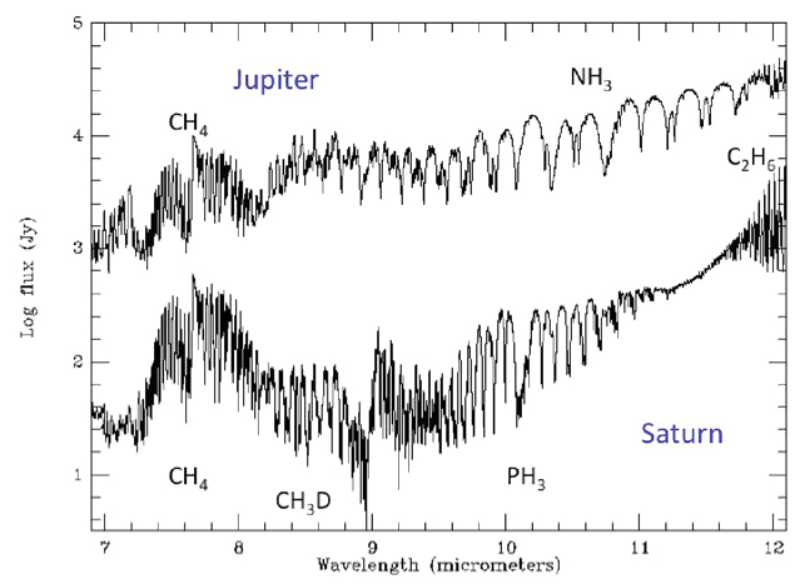

Figure 4. The spectra of Jupiter and Saturn, as observed in 1997 by the ISO

Short-Wavelength Spectrometer (SWS). Adapted from Encrenaz (2003). 
by the large abundance of phosphine $\left(\mathrm{PH}_{3}\right)$, a disequilibrium species which should not be observable according to thermochemical equilibrium models. Its presence in large abundances in Saturn's troposphere is the signature of strong upward motions which carry it from deep interiors (the same effect applies on Jupiter but to a weaker extent). A third difference between Jupiter and Saturn is the larger methane abundance in Saturn (for the reason mentioned above, see Section 5) which translates into a larger content of stratospheric hydrocarbons.

Another example can be found in the temperature maps of Uranus and Neptune, recorded at the VLT in 2006. Figure 5 mid-infrared maps of the two planets at $17 \mu \mathrm{m}$; this wavelength probes their tropospause. The map of Uranus is more or less symmetrical with respect to the equator, as expected for the present season since Uranus is close to equinox. Neptune, in contrast, exhibits a strong maximum at the south pole.

A possible explanation is that, since Neptune's southern summer solstice was in 2004, its south pole has been continuously illuminated for 40 years. This polar heating has an important consequence: gaseous methane, usually trapped in a cloud below the tropopause, can escape in the stratosphere and spread over the planet. This effect explains the anomalously high contents of methane and hydrocarbons in Neptune's stratosphere (Figure 6). Another difference between the two planets enhances this effect: just as Saturn with respect to Jupiter, Neptune shows a stronger vertical dynamical activity than Uranus, as shown by its active meteorology.

What could be the reason for these different dynamical behaviours? In the case of Uranus, the lack of atmospheric activity observed at the time of the Voyager encounter (1986) could have been due to the peculiar geometry of the planet, as its rotation axis was pointing toward the Sun. Indeed, we observed in 2005, just before equinox, some signs of meteorology (Figure 2). Another explanation, possibly connected with the previous one, is the lack of internal energy on Uranus, as compared to the other giants. In the case of Jupiter and Saturn, Voyager has measured an energy excess of 1.7 and 1.8 respectively, with respect to the absorbed solar energy; in the case of Neptune, the excess factor is 2.6 . The most plausible origin of this internal energy is the planet's contraction and cooling following the planet's accretion phase. Why is this mechanism not observed on Uranus? Possibly, convection is inhibited in Uranus' interior for some unknown reason.

\section{Conclusions and perspectives}

In spite of their common formation scenario in the outer solar system, each giant planet shows intrinsic properties which makes it very different from the others. Many

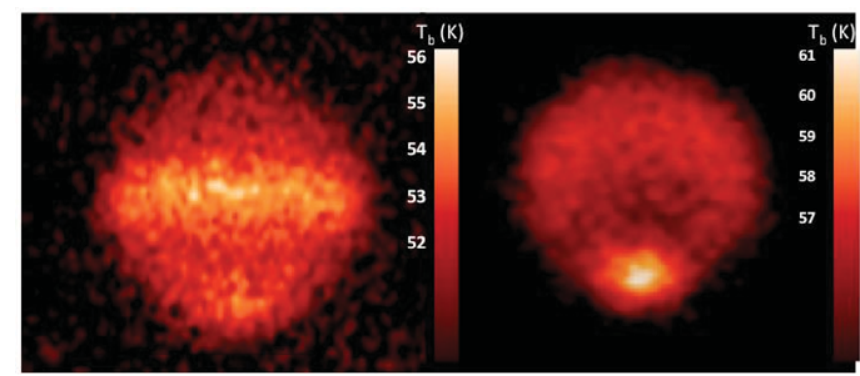

Figure 5. Mid-infrared maps $(17 \mu \mathrm{m})$ of Uranus and Neptune recorded in September 2006 with the VISIR instrument at the VLT. The $17 \mu \mathrm{m}$ wavelength probes the tropopause at a pressure level of about 100 mbar. Adapted from Orton et al., 2007. 
TEXES data

(arbitrary units)

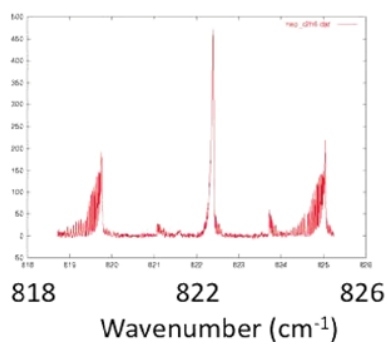

Synthetic model

$\mathrm{Q}\left(\mathrm{C}_{2} \mathrm{H}_{6}\right)=10^{-6}$

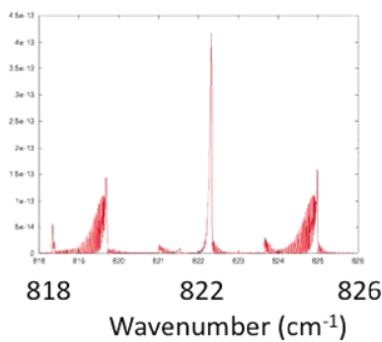

Figure 6. Infrared emission of ethane $\left(\mathrm{C}_{2} \mathrm{H}_{6}\right)$ on Neptune, observed with the TEXES instrument at IRTF in 2003. Left: TEXES data; right: synthetic model. These data illustrate the capabilities of ground-based infrared spectroscopy for studying the atmospheric composition of planets. ((c) T. Greathouse, J. Lacy and the TEXES Team).

pending questions still arise about their origin and evolution : What is the origin of the cold planetesimals which formed Jupiter? What is the enrichment in heavy elements in Saturn, Uranus and Neptune relative to hydrogen, as compared with their protosolar value? Did the giant planets migrate in the outer solar system, and by how much? If so, why did the migration stop? Why is Neptune more massive than Uranus, although at a larger heliocentric distance? Why is Uranus' rotation axis tilted to the ecliptic plane? Why are the internal structures of Uranus and Neptune different?

To answer these questions, new investigations are needed. Two ambitious missions are presently under study at NASA and ESA: The Europa and Jupiter System Mission (EJSM), which might be launched about 2020, and later the Titan and Saturn System Mission (TSSM) for horizon 2025. However, there are presently no plans to explore Uranus and Neptune, nor to send in-situ probes to measure the elemental composition of the giant planets. Still, these measurements are key diagnostics to understand the formation processes of these objects. This research is of special importance now, as it might help us to better understand the origin and history of the hundreds of exoplanets recently discovered around nearby stars.

\section{References}

Bagenal, F., Dowling, T., \& McKinnon, W. 2007, Jupiter: The planet, satellites and magnetosphere, Cambridge Planetary Science, Cambridge University Press

Bergstrahl, J. T., Miner, E. D., \& Matthews, M. S. 1990, Uranus, University of Arizona Press

Bézard, B. 2001, Neptune Encyclopedia of Astronomy and Astrophysics, P. Murdin, Edt. IoP Publishing, p.798

Cruikshank, D. P. 1995, Neptune and Triton, University of Arizona Press

Drossart, P. 2001, Saturn Encyclopedia of Astronomy and Astrophysics, P. Murdin, Edt. IoP Publishing

Encrenaz, T. 2001, Jupiter Encyclopedia of Astronomy and Astrophysics, P. Murdin, Edt. IoP Publishing, p. 1330

Encrenaz, T. 2003, Plan. Space Sci., 51, 89

Gehrels, T., \& Matthews, M. S. 1984, Saturn, University of Arizona Press

Herbert, F. 2001, Uranus and Neptune: Atmospheres, Ionospheres ad Magnetospheres, Encyclopedia of Astronomy and Astrophysics, P. Murdin, Edt. IoP Publishing, p. 3408

Lumine, J. I. 1993, The atmospheres of Uranus and Neptune, Ann. Rev. Astron. Astrophys., 31, 217 
Miner, E. D. 1998, Uranus - The Planet, Rings and satellites, John Wiley and Sons, New York Orton, G. S., Encrenaz, T., Leyrat, C., Puetter, R., \& Friedson, A. J. 2007, Astron. Astrophys, 473, L5

Owen, T., Mahaffy, P., Niemann, H. B., Atreya, S., Donahue, T., Bar Nun, A., \& de Pater, I. 1999, Nature, 402, 269

Owen, T. \& Encrenaz, T. 2006, Plan. Space Sci., 54, 1188

Smith, B. A. et al. 1986, Voyager 2 in the Uranian System: Results from the Imaging Team Science, 233, 97 